\title{
Research Square \\ The Relationship Between Gene Expression Pattern and the Severity of Asthma
}

\section{Shuang Lin}

People's Hospital of Xinjiang Uygur Autonomous Region

\section{Bin Luo}

The Fifth Afifiliated Hospital of Xinjiang Medical University

\section{Liping Cheng}

Xinjiang Medical University Affiliated Tumor Hospital

\section{Xiaohong Yang ( $\nabla 29852721 @ q q . c o m)$}

People's hospital of xinjiang Uygur autonomous region

\section{Research}

Keywords: Asthma, PPI network, KEGG, hub genes, STAT1

Posted Date: July 30th, 2020

DOI: https://doi.org/10.21203/rs.3.rs-46115/v1

License: (9) This work is licensed under a Creative Commons Attribution 4.0 International License. Read Full License 


\section{Abstract}

Background Because of heterogeneity, complexity of diagnosis and diversity of pathogenesis, the incidence and mortality of asthma are increased seriously. A structured and specific approach to assess and treat asthma may help clinicians.

Results A total of 838 common genes were found from quietness to exacerbation then to recovery of asthma. PPI network analysis identified 7 modules, and found 7 hub genes with high degree. Then, we verified the expression of hub genes in patients by quantitative real-time polymerase chain reaction (qRTPCR). Enrichment analysis and gene set enrichment analysis (GSEA) showed that exacerbation related genes were significantly related to immune and inflammatory response. Transcriptional regulators factor STAT1 had a significant regulatory effect on exacerbation related genes.

Conclusions These results indicated that seven hub genes were potential biomarkers and targets of asthma exacerbation. they were involved in the development of asthma through immune inflammatory signaling pathway. The results of this study not only provide a new research direction and theoretical basis for the exacerbation mechanism of asthma, but also provide a new target for clinical treatment.

\section{Introduction}

Asthma is a chronic inflammatory airway disease was characterized by heterogeneity, but there is no clear pathophysiological mechanism(Perlikos, et al. 2016). It has variable airway obstruction and bronchial hyperresponsiveness(Carr and Kraft 2017). Clinically, asthma patients will repeatedly appear wheezing, coughing, chest tightness and shortness of breath(Mims 2015). Due to the pathological unclear mechanism, the incidence and mortality of asthma are increasing in many parts of the world, making it a global health problem(Lemanske and Busse 1997). It is estimated that asthma will affect 334 million people in the world(To, et al. 2012). The prevalence of asthma varies widely from $0.2-21.0 \%$ in adults and from 2.8-37.6\% in children aged 6 to 7 years(Aaron, et al. 2018; Papi, et al. 2018).

Asthma is the result of complex gene environment interaction(Papi, et al. 2018). Therefore, the disease mechanism is complicated and diverse. Theoretically, many cytokines and chemokines may be related to the endogenous "neutrophil rich" and "eosinophil rich" of asthma(Wenzel 2012). More and more evidence showed that there were two broad inflammatory phenotypes in the airway of asthmatic patients: eosinophilic inflammation and other neutrophil dominated inflammation(Gibson, et al. 2001). Recent studies had highlighted the increased response of Th1 / IFN-y and Th17 / IL-17 in asthma, which was often associated with neutrophil infiltration in the airway (Raundhal, et al. 2015; Ray, et al. 2016).

In the investigation, extensive clinical evaluation and testing were often impractical(Kirenga, et al. 2019). Accurate diagnosis of asthma is important because it will contribute to enhancing the incidence and mortality of this disease. Despite drug therapy recommended by national and international asthma guidelines, asthma control is often poor(Chung, et al. 2014; National Asthma and Prevention 2007). The evaluation and diagnosis of asthma should first have a comprehensive history and physical 
examination(Winders, et al. 2019). Other parts include lung function, medical level, eosinophil count and allergy test (Boulet, et al. 2019; Bousquet, et al. 2010; Singh, et al. 2019).

Asthma is sometimes difficult to treat, resulting in a disproportionate cost to the medical system (Jones, et al. 2018). The objectives of asthma treatment include prevention of recurrent exacerbations (Fuhlbrigge, et al. 2012). However, gave the heterogeneity of the disease, a "one size fits all" treatment strategy seems no longer suitable for effectively pursuing these goals. At present, a variety of biomarkers can identify the characteristics of high endogenous inflammation in adults and children with asthma, and targeted treatment research has been carried out in (Galeone, et al. 2018; Uwaezuoke, et al. 2018). IL-17 pathway may be the target of severe asthma disease control (Al-Ramli, et al. 2009; Suzuki, et al. 2016). Therefore, it is extremely important to determine the corresponding biomarkers according to the exacerbation and recovery of asthma. In this study, we will identify biomarkers and potential molecular mechanisms of asthma exacerbation based on the expression patterns of genes during exacerbation and recovery of asthma patients.

\section{Results}

\section{Systemic molecules in the process of asthma attack and recovery}

To identify which factors were involved in the process of asthmatic patients, we analyzed the transcriptome data of GSE19301 data. Compared with the quiet group, Toll like receptor and NOD like receptor signaling pathway in the exacerbation group were activated (Fig. 1A). Compared with the followup group, NOD like receptor signaling pathway and apoptosis in the exacerbation group were activated (Fig. 1B). The results showed that there were the same regulatory mechanism and different molecular changes in the process of asthma from quiet to exacerbation to follow-up. Therefore, we compared the gene expression of three groups. There were 2194 differentially expressed genes (DEGs) between exacerbation and quiet groups (Fig. 1C). There were 1376 DEGs between exacerbation and follow-up groups (Fig. 1D). These results indicated that some genes and signaling pathways played crucial roles in the process of asthma from tranquility to exacerbation and then to tranquility.

\section{Identifying asthma-associated PPI modules from DEGs}

To further explore the interactions of differentially expressed genes in the process of exacerbation to recovery, we mapped the common genes in the two groups of DEGs into PPI network. 838 common genes were mapped into PPI network. Next, the module searching procedure identified 7 modules (Figure S1). The distribution of the genes with the highest or lowest expression level of exacerbation group in the module compared with quiet or follow-up was shown by heatmap (Fig. 2A). Up regulated genes of exacerbation and quiet were all in M2, and the down regulated genes were all in M5, respectively (Fig. 2B). Similarly, the up regulated genes of exacerbation and follow-up were all in $\mathrm{M} 2$, and the down regulated 
genes were all in M5 (Fig. 2C). Therefore, M2 genes may promote asthma, while M5 genes may inhibit asthma.

On the other hand, hub gene of each module by the degree in the module was screened (Table 2). Depending on the expression of hub genes in three groups of asthma, ENO1, OAS3 and TLR4 were the highest expressed in exacerbation, while HSP90AB1, HSPA9, PTCD3 and UBA52 were the lowest expressed in exacerbation (Fig. 2D). Therefore, they are considered to be potential genes for positive or reverse regulation of asthma exacerbation. Importantly, we verified the expression of these key genes during the exacerbation of asthma by qPCR (Fig. 2E). In addition, the results of correlation analysis also proved that there was a significant negative correlation between high or low expression genes in exacerbation (Fig. 2F). These results suggested that key genes can regulate the exacerbation of asthma, which may be used as a biomarker of asthma exacerbation for further study.

Table 2: The hub genes of modules for the disorder gene in the process of asthma deterioration to recovery

\begin{tabular}{|ll|}
\hline Module & Symbol \\
\hline $\mathrm{m} 1$ & UBA52 \\
\hline $\mathrm{m} 2$ & OAS3 \\
\hline $\mathrm{m} 3$ & TLR4 \\
\hline $\mathrm{m} 4$ & HSP90AB1 \\
\hline $\mathrm{m} 5$ & PTCD3 \\
\hline $\mathrm{m} 6$ & ENO1 \\
\hline $\mathrm{m} 7$ & HSPA9 \\
\hline
\end{tabular}

\section{Biological function and signal pathway involved in the process of asthma}

To identify the biological functions and signaling pathways involved in the progression of asthma, module genes were enriched and analyzed. A total of 1741 BP, 113 CC, 232 MF and 65 KEGG signaling pathways were enriched by the module genes. Among them, genes mainly enriched in activation of the innate immune response and other immune inflammatory functions (Fig. 3A, B, C). Also enriched in Toll like receptor signaling pathway, regulation of cellular amine metallic process signal pathways (Fig. 3D). In the up-regulated or down-regulated pathway, we found that NOD - like receptor signaling pathway was activated in exacerbation group, but decreased in quiet and follow-up (Fig. 3E, F). ENO1, OAS3 and TLR4 were mainly enriched in coenzyme metallic process, nucleotidyltransferase activity, regulation of inflammatory response and others. HSP90AB1, HSPA9, PTCD3 and UBA52 were mainly involved in 


\section{Transcription factors (TF) regulating dysfunctional molecules}

Transcription regulation played an important role in the progress of diseases. Since we had observed that the module genes were associated with the exacerbation and recovery of asthma. We obtained 76 transcription factor regulatory module genes (Fig. 4A). The results of correlation analysis identified the transcription factors STAT1 was highly correlated with the module genes, including XAF1, PSMB9, IFIT3 and IRF7 (Fig. 4B). Therefore, we believed that STAT1 was an important regulatory role in the pathogenesis of asthma.

\section{Discussion}

The pathogenesis of asthma is not completely clear(Daya, et al. 2019). At present, the diagnosis and treatment of asthma is also challenging. Therefore, this study is to explore the gene expression patterns in the process of asthma. In order to identify biomarkers that can diagnose the exacerbation of asthma and the mechanism of exacerbation with potential therapeutic value.

The differentially expressed genes were thought to represent the development of asthma(Pascoe, et al. 2017). Interestingly, we found that the top six genes up regulated from quiet to exacerbation were the top six genes down regulated in follow up group. Among them, the expression of SIGLEC-1 in lung tissue was specific to alveolar macrophages, and the single nucleotide polymorphisms in SIGLEC-1 was related to the severity of asthma(Souza de Lima, et al. 2017). CCL2 expression was enhanced in allergic asthma induced by OVA sensitized mice (Jiang, et al. 2019). The expression of genes in asthma patients with exacerbation ran counter to that in patients with recovery, they may be indication of exacerbation, as well as potential therapeutic target.

The genes in each module may represent a mechanism of asthma. Enrichment analysis showed that genes in M2 were mainly related to activation of innate immune response, Toll like receptor signaling pathway. Asthma was often considered as the result of abnormal activation of Th2 to environmentally sound allergens(Thiriou, et al. 2017). Toll like receptor signaling pathway was activated in patients in the exacerbation group. In fact, the complex interaction between environmental allergens and TLRs results in the release of various proinflammatory mediators by innate cells supporting the development of asthma(Michels, et al. 2018; Zakeri and Yazdi 2017). Genes in M5 were mainly enriched in regulation of cellular amine metallic process and other biological functions. This may be related to the metabolism of asthma after drug treatment(Cahill, et al. 2017; Peters and Busse 2017). In addition, some allergens interact with NOD like receptors on airway epithelial cells, and act as "danger signals" to induce asthma (Theofani, et al. 2019). 
In the hub genes, TLR4 was reported to be significantly associated with the occurrence and development of asthma (Hwang, et al. 2019). Heat shock protein (HSP) played a major role in promoting inflammation and anti-inflammatory response(Hallenbeck and Kaplan 1987). Heat shock factor 1 (HSF 1) could attenuate airway hyperresponsiveness and airway inflammation in asthmatic mice (Wang, et al. 2017). In general, the highly expressed hub gene was mainly enriched in the inflammatory response. There was an inseparable relationship between inflammation and asthma(Denlinger, et al. 2017; Hu 2017). The low expression of hub genes was mainly concentrated in response to interleukin-4 (IL-4) and interleukin-12 (IL-12). IL-4 played a key role in type 2 asthma, and could reduce the exacerbation rate of asthma after blocking(Castro, et al. 2018; Parulekar, et al. 2018). The levels of IL-4 and IL-12 in the peripheral blood of the patients were related to the pulmonary function, cellular immune function and quality of life of children with moderate and severe asthma (Cui, et al. 2017).

In this study, we also predicted the regulator of the module gene of asthma. STAT1, a transcription factor with a high correlation with module genes. Studies had shown that STAT1 was associated with asthma exacerbation (Gomez, et al. 2018; Su, et al. 2016). We believed that STAT1 could be involved in the exacerbation and recovery of asthma by regulating target genes.

The pathophysiology of asthma was complicated. There was no single cytokine related to the whole pathogenesis of asthma. Despite the best guidelines for treatment, and regardless of the severity of the underlying disease, asthma patients will experience exacerbation, which is caused by aggravation of the existing inflammatory process and the loss of disease control (Castillo, et al. 2017). Our results showed that many inflammatory cytokines were involved in the innate and adaptive immunity of asthma. In particular, hub genes we identified may serve as a biomarker of asthma exacerbation and a potential target for treatment.

\section{Conclusions}

PPI network was used to analyze the key genes of asthma exacerbation, and 7 hub genes were identified to be related to asthma exacerbation. Molecular mechanism analysis showed that these genes participate in the development of asthma through the signal pathway of immune inflammation. Transcription regulators predicted that STAT1 had a significant regulatory effect on exacerbation related genes.

\section{Materials And Methods}

\section{Datasets}

We collected the dataset GSE19301 from GEO database. which includes quiet (394 samples), exacerbation (166 samples) and follow-up (125 samples) (Bjornsdottir, et al. 2011). Series matrix files and clinical information tables were downloaded from the GEO website. 


\section{Identification of differentially expressed genes}

The "sva" R package was used to conduct batch normalization of the expression data from the three different datasets. Then, the normalized expression matrix of quiet, exacerbation and follow-up differences were analyzed by "limma" $\mathrm{R}$ package respectively. The differentially expressed genes were obtained by setting threshold $p<0.05$.

\section{Constructing protein protein interaction (PPI) network and identify modules}

Download the protein interaction data from the protein interaction database (String). Interaction data containing only differential genes was screened, and the score $>500$ was set. For the analysis of the recognition module, we first import the selected protein interaction into the software of Cytoscape. We used the ClusterONE plug-in for module mining. Then, network analysis of gene subnets in the module was carried out, and the degree of nodes was obtained. The higher the degree of a node, the more important it is, the more likely it is to represent the characteristics of the module.

\section{Patients}

Whole blood samples were collected from patients with asthma, including 3 quiet, 3 exacerbation and 3 follow-up, respectively. The human study protocol, consent form, and consent procedure were approved by the medical ethic committee of Xinjiang Medical University. All patients were informed and gave their written consent to have their blood for research purpose. This protocol complied with the Code of Ethics of the World Medical Association (Declaration of Helsinki) and in agreement with ethical guidelines set by China law.

\section{Quantitative real-time polymerase chain reaction}

To isolate RNA, $200 \mu \mathrm{L}$ human blood samples were collected from patients and mix well with $1 \mathrm{ml}$ of Trizol reagent (Invitrogen, USA), according to the manufacturer's instructions. After quantification, $0.5 \mu \mathrm{g}$ of RNA was reversed transcribed with SuperScriptIII (Invitrogen, USA). Quantitative real-time polymerase chain reaction (qRT-PCR) was performed on Bio-Rad CFX96 with SYBR Master Mix (Invitrogen, USA). All samples were normalized to the signal generated from GAPDH. Sequences of primers used in this study are listed in Table 1. The experiments were repeated at least 3 times, and results were analyzed with analysis of variance. Data are shown as fold change $(2-\Delta \Delta \mathrm{Ct})$. Statistical analysis was performed using SPSS software and student's $t$ test. $P<0.05$ was considered statistically significant. 
Table 1

Primer sequences used for qPCR.

\begin{tabular}{|c|c|c|}
\hline Gene & \multicolumn{2}{|c|}{ Oligonucleotide Sequence } \\
\hline \multirow[t]{2}{*}{ UBA52 } & Forward & 5' GCGTCCCAAGAAGAAGGTCA 3' \\
\hline & Reverse & 5' ACCAATTGCTGCTCCAGTCA 3' \\
\hline \multirow[t]{2}{*}{ OAS3 } & Forward & 5' GCGATGCCCGCATCTCACTG 3' \\
\hline & Reverse & 5' CAGTGAGATGCGGGCATCGCC 3' \\
\hline \multirow[t]{2}{*}{ TLR4 } & Forward & 5' CTACCTGGAATGGGAGGACA 3' \\
\hline & Reverse & 5' CTTAGCAGCCATGTGTTCCA 3' \\
\hline \multirow[t]{2}{*}{ HSP90AB1 } & Forward & 5' TTGACATCATCCCCAACCCTC 3' \\
\hline & Reverse & 5' ACCAAACTGCCCAATCATGGA 3' \\
\hline \multirow[t]{2}{*}{ PTCD3 } & Forward & 5' ACATGGGATAAAGTGGCTGTTCT 3' \\
\hline & Reverse & 5' GACTCCAGAGCAGAGGTTGG 3' \\
\hline \multirow[t]{2}{*}{ EN01 } & Forward & 5' CCCAGUGGUGUCUAUCGAATT 3' \\
\hline & Reverse & 5' UUCGAUAGACACCACUGGGTT 3' \\
\hline \multirow[t]{2}{*}{ HSPA9 } & Forward & 5' CTTGTTTCAAGGCGGGATTATGC 3' \\
\hline & Reverse & 5' ACACCATGTATTCCGGGTCAAG 3' \\
\hline \multirow[t]{2}{*}{ GADPH } & Forward & 5' GCATCCTGGGCTACACT 3' \\
\hline & Reverse & 5' CACCACCCTGTTGCTGT 3' \\
\hline
\end{tabular}

\section{Enrichment and gene-set enrichment analysis (GSEA)}

To clarify the possible biological roles of these genes in PPI networks, the cluster Profiler R package was used to create KEGG enrichment paths, results and plots. $P<0.05$ indicated that KEGG pathway had significant difference.

We performed gene set enrichment analysis using the application available at the Broad Institute Gene Set Enrichment Analysis website. We tested the KEGG pathways gene sets. Activation or inhibition of KEGG was evaluated by Gene Set Variation Analysis (GSVA) algorithm.

\section{Prediction of regulatory transcription factors for modular genes}


To explore the pivotal regulators of module genes in the exacerbation and recovery of asthma, all human transcription factor target data was used in TRRUST V2 database as a background set for pivot analysis(Daya, et al. 2019; Han, et al. 2018). Setting threshold $P<0.05$.

\section{Declarations}

\section{Ethics approval and consent to participate}

This protocol complied with the Code of Ethics of the World Medical Association (Declaration of Helsinki) and in agreement with ethical guidelines set by China law.

\section{Consent for publication}

All authors agree to publish.

\section{Acknowledgements and Funds}

The work was supported by the Natural Science Foundation of Xinjiang Uygur Autonomous Region (No. 2016D01C112).

\section{Availability of data and materials}

Not applicable

\section{Competing interests}

All authors declare no competing interests.

\section{Authors' contributions}

Yunwen Cui supervised the data analysis and interpretation. Bin Luo contributed to data and manuscript preparation. Liping Cheng and Xiaohong Yang conceived and designed the study, supervised the experimental work, data analysis and interpretation, and manuscript critical review.

\section{References}

1. Shawn, D. A., Louis, P. B., Helen, K. R. et al. Underdiagnosis and Overdiagnosis of Asthma. Am J Respir Crit Care Med. 2018; 198:1012-1020; doi:10.1164/rccm.201804-0682Cl. 
2. Wisam, A., David, P., Fazila C. et al. T(H)17-associated cytokines (IL-17A and IL-17F) in severe asthma. J Allergy Clin Immunol. 2009; 123:1185-7; doi:10.1016/j.jaci.2009.02.024.

3. Bjornsdottir, U. S., Holgate, S. T., Reddy, P. S. et al. Pathways activated during human asthma exacerbation as revealed by gene expression patterns in blood. PLoS One 2011; 6:e21902; doi:10.1371/journal.pone.0021902.

4. Boulet, L. P., Reddel, H. K., Bateman, E. et al. The Global Initiative for Asthma (GINA): 25 years later. Eur Respir J. 2019; 54(2). doi:10.1183/13993003.00598-2019.

5. Bousquet, J., Mantzouranis, E., Cruz, A. A. et al. Uniform definition of asthma severity, control, and exacerbations: document presented for the World Health Organization Consultation on Severe Asthma. J Allergy Clin Immunol. 2010; 126:926-38. doi:10.1016/j.jaci.2010.07.019.

6. Cahill, K. N., Katz, H. R., Cui, J., et al. KIT Inhibition by Imatinib in Patients with Severe Refractory Asthma. N Engl J Med. 2017; 376(20):1911-1920; doi:10.1056/NEJMoa1613125.

7. Carr, T. F., and M. Kraft. Management of Severe Asthma before Referral to the Severe Asthma Specialist. - J Allergy Clin Immunol Pract. 2017; 5:877-886; doi:10.1016/j.jaip.2017.04.027.

8. Castillo, J. R., Peters, S. P., Busse, W. W. Asthma Exacerbations: Pathogenesis, Prevention, and Treatment. J Allergy Clin Immunol Pract. 2017; 5:918-927; doi:10.1016/j.jaip.2017.05.001.

9. Castro, M., Corren, J., Pavord, I. D. et al. Dupilumab Efficacy and Safety in Moderate-to-Severe Uncontrolled Asthma. N Engl J Med. 2018; 378:2486-2496; doi:10.1056/NEJMoa1804092.

10. Chung, K. F., Wenzel, S. E., Brozek, J. L. et al. International ERS/ATS guidelines on definition, evaluation and treatment of severe asthma. Eur Respir J. 2014; 43:343-73; doi:10.1016/j.jaip.2017.05.001.

11. Cui, A. I., Zhao, J., Liu, S. X. et al. Associations of IL-4, IL-6, and IL-12 levels in peripheral blood with lung function, cellular immune function, and quality of life in children with moderate-to-severe asthma. Medicine (Baltimore). 2017; 96:e6265; doi:10.1097/MD.0000000000006265.

12. Daya, M., Rafaels, N., Brunetti, T. M. al. Association study in African-admixed populations across the Americas recapitulates asthma risk loci in non-African populations. Nat Commun. 2019; 10:880; doi:10.1038/s41467-019-08469-7.

13. Denlinger, L. C., Phillips, B. R., Ramratnam, S. et al. Inflammatory and Comorbid Features of Patients with Severe Asthma and Frequent Exacerbations. Am J Respir Crit Care Med. 2017. 195:302-313; doi:10.1164/rccm.201602-04190C.

14. Fuhlbrigge, A., Peden, D., Apter, A. J. et al. Asthma outcomes: Exacerbations. Journal of Allergy and Clinical Immunology. 2012;129:S34-S48.

15. Galeone, C., Scelfo, C., Bertolini, F. et al. Precision Medicine in Targeted Therapies for Severe Asthma: Is There Any Place for "Omics" Technology? Biomed Res Int. 2018; 4617565; doi:10.1155/2018/4617565.

16. Gibson, P. G., Simpson, J. L., Saltos, N. Heterogeneity of airway inflammation in persistent asthma : evidence of neutrophilic inflammation and increased sputum interleukin-8. Chest. 2001. 119:1329-36; doi:10.1378/chest.119.5.1329. 
17. Gomez, J. L., Diaz M. P., Nino, G. Impaired type I interferon regulation in the blood transcriptome of recurrent asthma exacerbations. BMC Med Genomics. 2018;11:21; doi:10.1186/s12920-018-0340-3.

18. Hallenbeck, P. L., and Kaplan, S. Cloning of the gene for phosphoribulokinase activity from Rhodobacter sphaeroides and its expression in Escherichia coli. J Bacteriol. 1987; 169:3669-78; doi:10.1128/jb.169.8.3669-3678.

19. Han, J., Cho, J. W., Lee, S. Y. et al. TRRUST v2: an expanded reference database of human and mouse transcriptional regulatory interactions. Nucleic Acids Res. 2018; 46:D380-D386; doi:10.1093/nar/gkx1013.

20. Hu, D. Role of Anti-inflammatory Cytokines IL-35 and IL-37 in Asthma. - Inflammation. 2017; 40:697707; doi:10.1007/s10753-016-0480-6.

21. Hwang, Y. H., Lee, Y. J., Paik, M. J. et al. Inhibitions of HMGB1 and TLR4 alleviate DINP-induced asthma in mice. Toxicol Res (Camb). 2019; 8:621-629; doi:10.1039/c9tx00048h.

22. Jiang, S., Wang, Q., Wang, Y. X. et al. Blockade of CCL2/CCR2 signaling pathway prevents inflammatory monocyte recruitment and attenuates OVA-Induced allergic asthma in mice. Immunol Lett. 2019; 214:30-36; doi:10.1016/j.imlet.2019.08.006.

23. Jones, T. L., Neville, D. M., Chauhan, A. J. Diagnosis and treatment of severe asthma: a phenotypebased approach. Clin Med (Lond). 2018; 18:s36-s40; doi:10.7861/clinmedicine.18-2-s36.

24. Kirenga, B. J., Jong, C. D., Katagira, W. Prevalence and factors associated with asthma among adolescents and adults in Uganda: a general population based survey. BMC Public Health. 2019; 19:227; doi:10.1186/s12889-019-6562-2.

25. Lemanske, R. F., Busse, W. W. Asthma. JAMA. 1997; 278:1855-73.

26. Michels, K. R., Lukacs, N. W., Fonseca, W. TLR Activation and Allergic Disease: Early Life Microbiome and Treatment. Curr Allergy Asthma Rep. 2018; 18:61; doi:10.1007/s11882-018-0815-5.

27. Mims, J. W. Asthma: definitions and pathophysiology. Int Forum Allergy Rhinol. 2015. Suppl 1:S2-6.

28. National Asthma, Education, and Program Prevention. Expert Panel Report 3 (EPR-3): Guidelines for the Diagnosis and Management of Asthma-Summary Report. J Allergy Clin Immunol. 2007; 120:S94138; doi:10.1016/j.jaci.2007.09.043.

29. Papi, A., Brightling, C., Pedersen, S. E. et al. Asthma. Lancet. 2018; 391:783-800; doi:10.1016/S01406736(17)33311-1.

30. Parulekar, A. D., Kao, C. C., Diamant Z. et al. Targeting the interleukin-4 and interleukin-13 pathways in severe asthma: current knowledge and future needs. Curr Opin Pulm Med. 2018. 24:50-55. doi:10.1097/MCP.0000000000000436.

31. Pascoe, C. D., Obeidat, M., Arsenault, B. A. et al. Gene expression analysis in asthma using a targeted multiplex array. BMC Pulm Med. 2017; 17:189; doi:10.1186/s12890-017-0545-9.

32. Perlikos, F., Hillas, G., Loukides, S. Phenotyping and Endotyping Asthma Based on Biomarkers. Curr Top Med Chem. 2016; 16:1582-6; doi:10.2174/1568026616666150930120803. 
33. Peters, S. P., and Busse, W. W. New and Anticipated Therapies for Severe Asthma. J Allergy Clin Immunol Pract. 2017; 5:S15-S24; doi:10.1016/j.jaip.2017.07.008.

34. Raundhal, M., Morse, C., Khare, A. et al. High IFN-gamma and low SLPI mark severe asthma in mice and humans. J Clin Invest. 2015; 125:3037-50; doi:10.1172/JCI80911.

35. Ray, A., Raundhal, M., Oriss, T. B. et al. Current concepts of severe asthma. J Clin Invest. 2016; 126:2394-403; doi:10.1172/JCl84144.

36. Singh, D., Agusti, A., Anzueto, A. et al.Global Strategy for the Diagnosis, Management, and Prevention of Chronic Obstructive Lung Disease: the GOLD science committee report 2019. Eur Respir J. 2019; 53(5); doi:10.1183/13993003.00164-2019.

37. Souza, D. L. D., Nunes, V. C. L., Ogusku, M, M. et al. Polymorphisms in SIGLEC1 contribute to susceptibility to pulmonary active tuberculosis possibly through the modulation of IL-1ss. Infect Genet Evol. 2017; 55:313-317; doi:10.1016/j.meegid.2017.09.031.

38. Su, X., Pan, J., Bai, F. et al. IL-27 attenuates airway inflammation in a mouse asthma model via the STAT1 and GADD45gamma/p38 MAPK pathways. J TransI Med. 2016; 14:283;doi:10.1186/s12967016-1039-x.

39. Suzuki, Y., Maazi, H., Sankaranarayanan, I. et al. Lack of autophagy induces steroid-resistant airway inflammation. - J Allergy Clin Immunol. 2016; 137:1382-1389 e9. doi:10.1016/j.jaci.2015.09.033.

40. Theofani, E., Semitekolou, M., Morianos, I. et al. Targeting NLRP3 Inflammasome Activation in Severe Asthma. J Clin Med. 2019; 8(10); doi:10.3390/jcm8101615.

41. Thiriou, D., Morianos, M., Xanthou, G. et al. Innate immunity as the orchestrator of allergic airway inflammation and resolution in asthma. Int Immunopharmacol. 2017; 48:43-54; doi:10.1016/j.intimp.2017.04.027

42. To, T., Stanojevic, S., Moores, G. et al. Global asthma prevalence in adults: findings from the crosssectional world health survey. BMC Public Health. 2012; 12:204; doi:10.1186/1471-2458-12-204.

43. Uwaezuoke, S. N., Ayuk, A. C., Eze, J. N. Severe bronchial asthma in children: a review of novel biomarkers used as predictors of the disease. J Asthma Allergy. 2018; 11:11-18; doi:10.2147/JAA.S149577.

44. Wang, J., Xin, L. H., Cheng, W. et al. [Effect of heat shock factor 1 on airway hyperresponsiveness and airway inflammation in mice with allergic asthma]. - Zhongguo Dang Dai Er Ke Za Zhi. 2017; 19:222228.

45. Wenzel, S. E. Asthma phenotypes: the evolution from clinical to molecular approaches. Nat Med. 2012; 18:716-25;doi:10.1038/nm.2678.

46. Winders, T. A., Wilson, A. M., Fletcher, M. J. et al. A Patient-Centered Description of Severe Asthma: Patient Understanding Leading to Assessment for a Severe Asthma Referral (PULSAR). - Patient. 2019; 12:539-549; doi:10.1007/s40271-019-00371-0.

47. Zakeri, A., Yazdi, F. G. Toll-like receptor-mediated involvement of innate immune cells in asthma disease. Biochim Biophys Acta Gen Subj. 2017; 1861:3270-3277; doi:10.1016/j.bbagen.2016.08.009. 

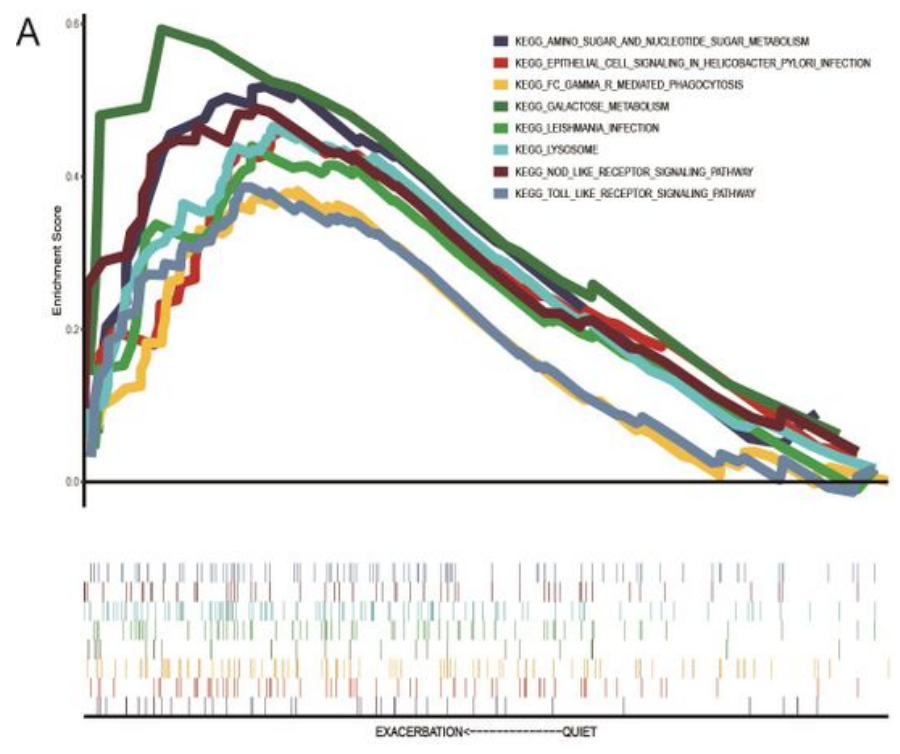

C

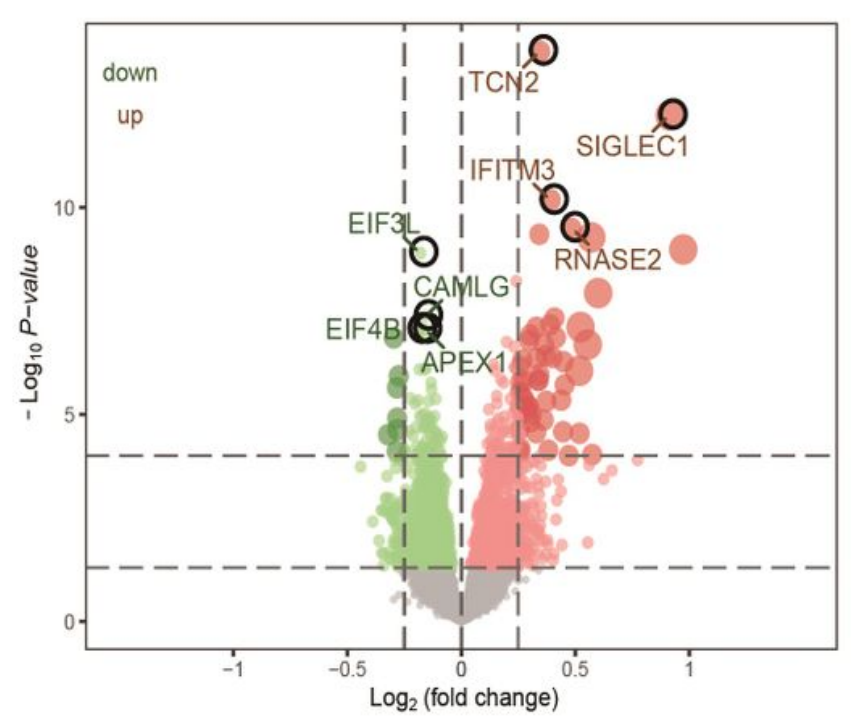

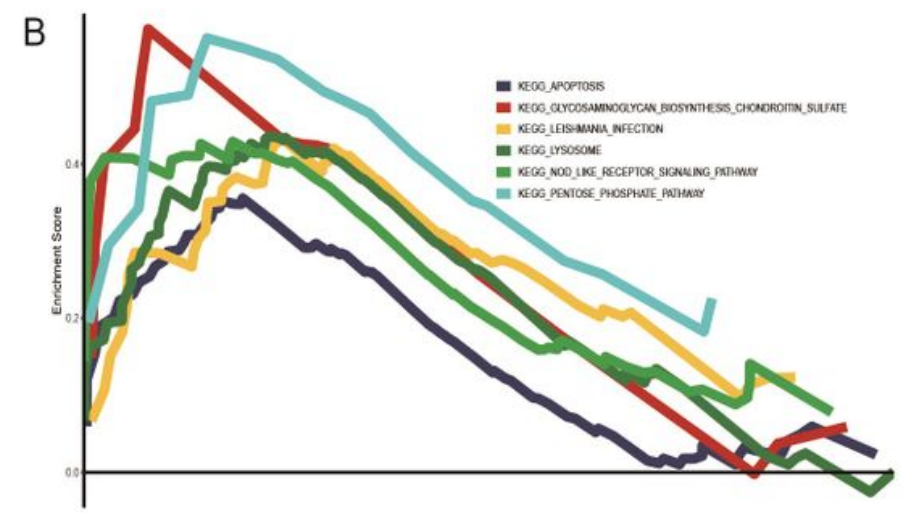

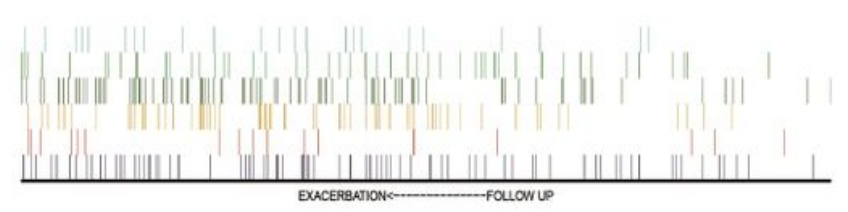

EXACERBATION

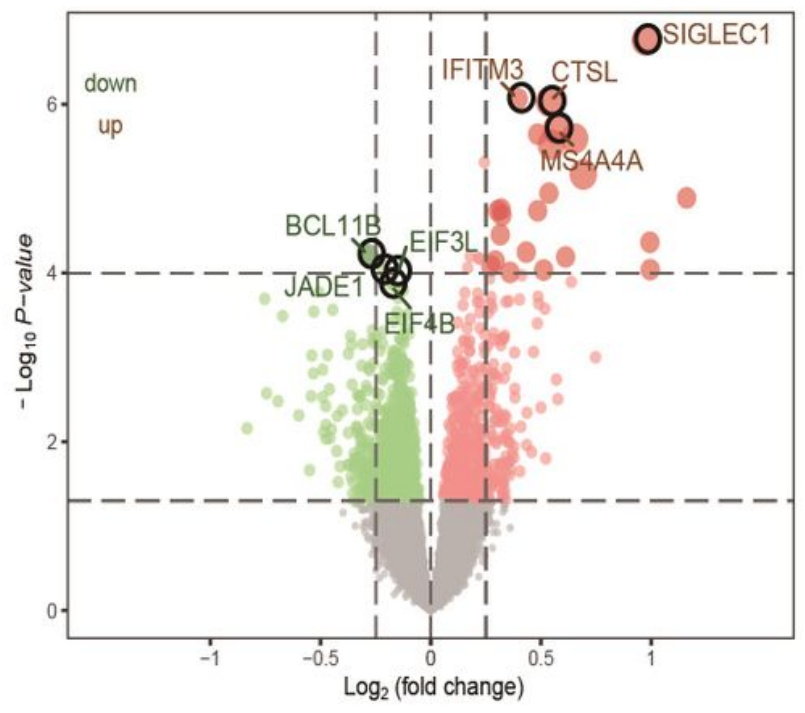

Figure 1

Gene expression and signal transduction from an asthma attack to recovery. A. KEGG signaling pathway activated during asthma exacerbation. B. KEGG signaling pathway inhibited during the course of asthma exacerbation to recovery. C. Compared with patients with asthma at quiet, the differentially expressed genes in the patients with exacerbation. D. Compared with the follow up of asthmatic patients, the differential expression of genes in patients with exacerbation. Red node represents up regulated gene, blue node represents down regulated gene. 

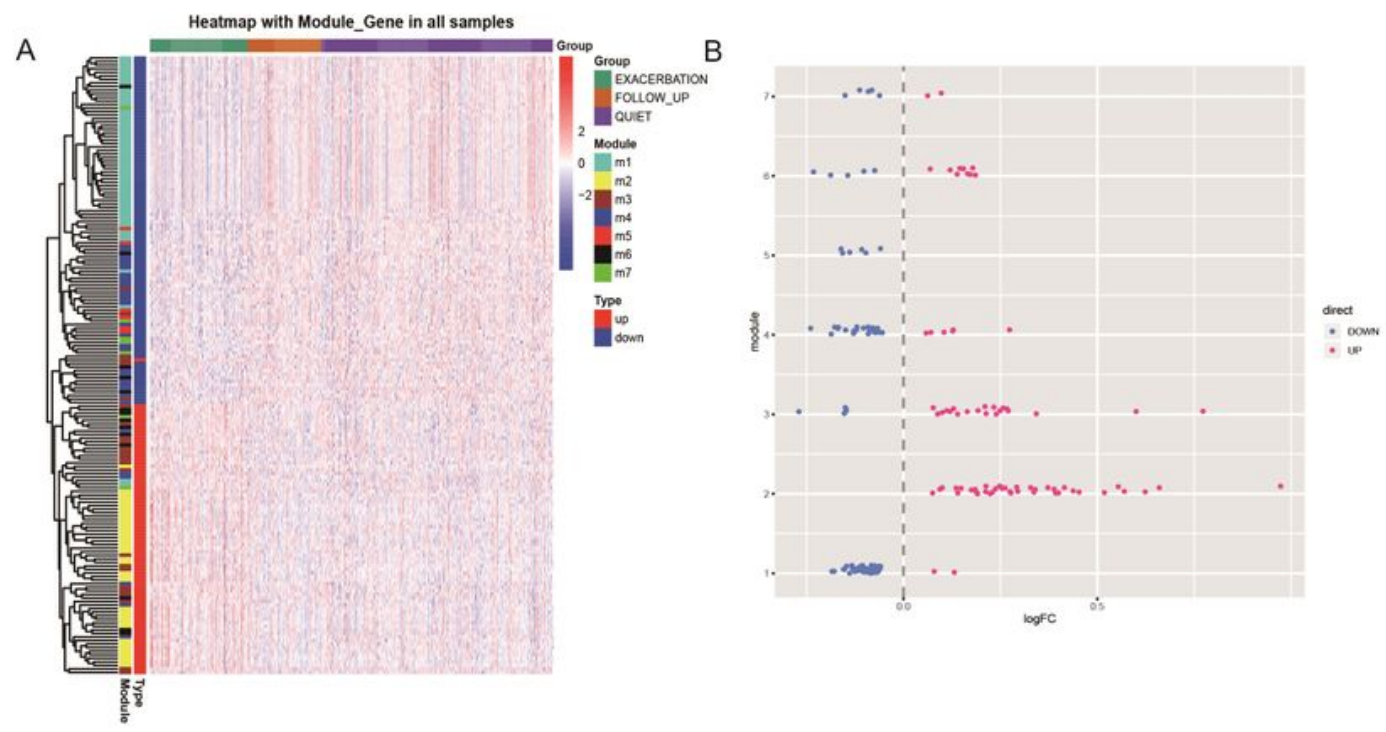

C

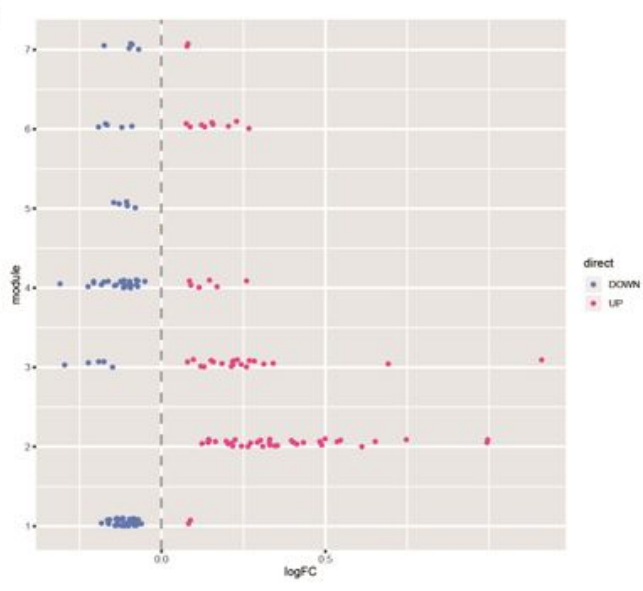

E

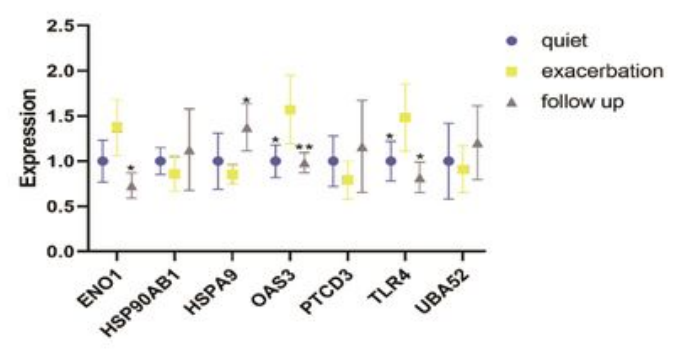

D

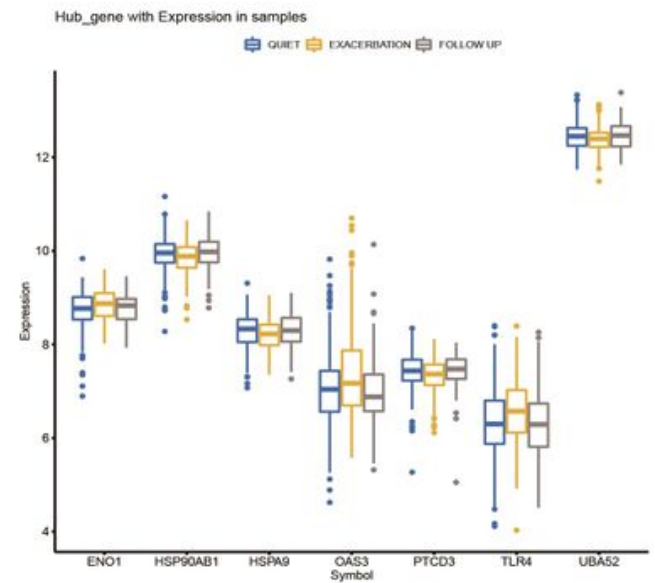

F

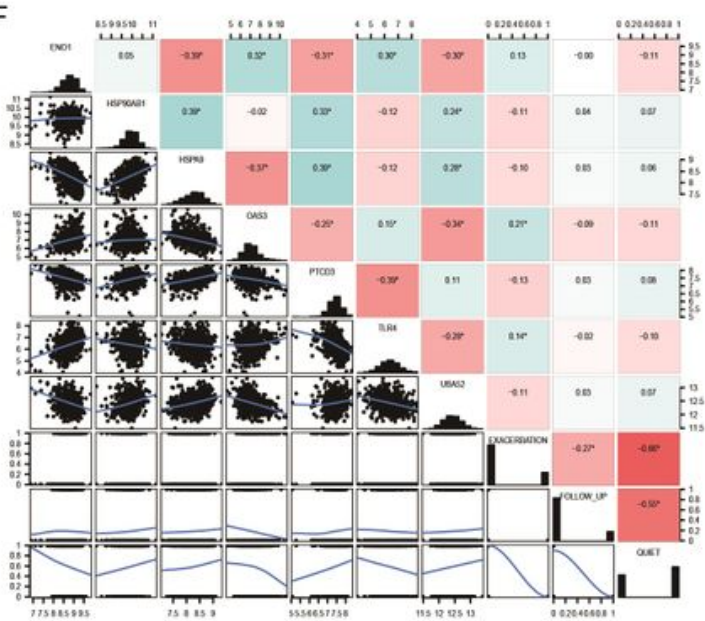

Figure 2

Key genes recognition of maladjusted molecules in the process of asthma exacerbation and recovery. A. The expression of the intersection genes for two groups in modules was demonstrated by thermogram. The red node represents the genes up regulated in two groups, and the blue node represents the genes down regulated in two groups. In the module, the up regulation or down-regulation genes of exacerbation compared with quiet (B) and the up regulation or down regulation genes of exacerbation compared with 
follow-up (C). D. The expression of each module's hub gene in quiet, exacerbation and follow up groups in dataset. E. The expression of hub genes in blood of quiet, exacerbation and follow up patients. ${ }^{*} \mathrm{P}<0.05$, $\star \star P<0.01$ compared to exacerbation. $F$. The correlation between module hub genes and between genes and phenotypes.
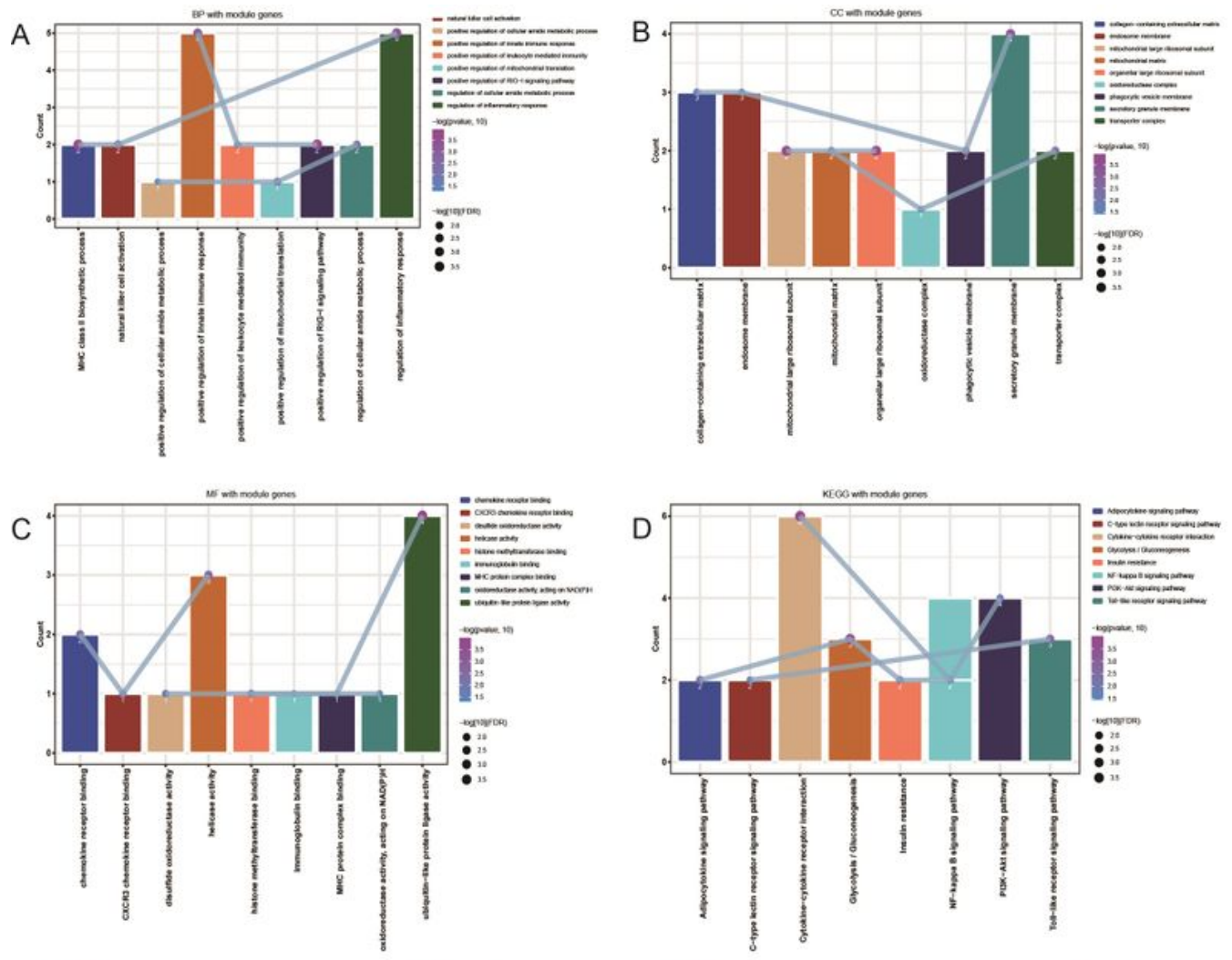

E
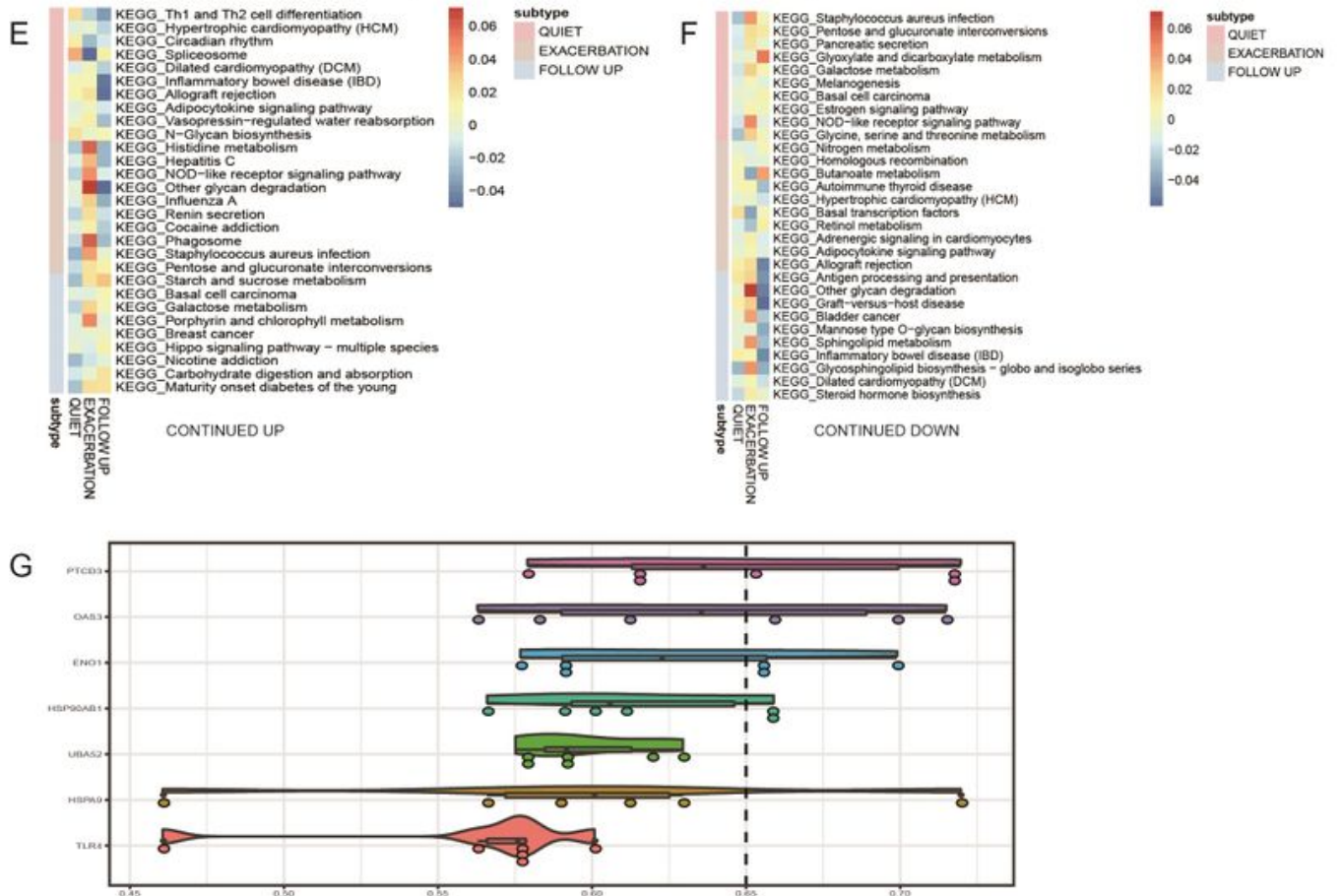

Figure 3 
Enrichment analysis of module genes. The BP (A), CC (B) and MF (C) of module genes enrichment. D. The KEGG signaling pathway of module gene enrichment. GSVA identifies signaling pathways that are continuously up-regulated (E) or down regulated in three states (F). G. Raincloud map of functional similarity between key genes.

A

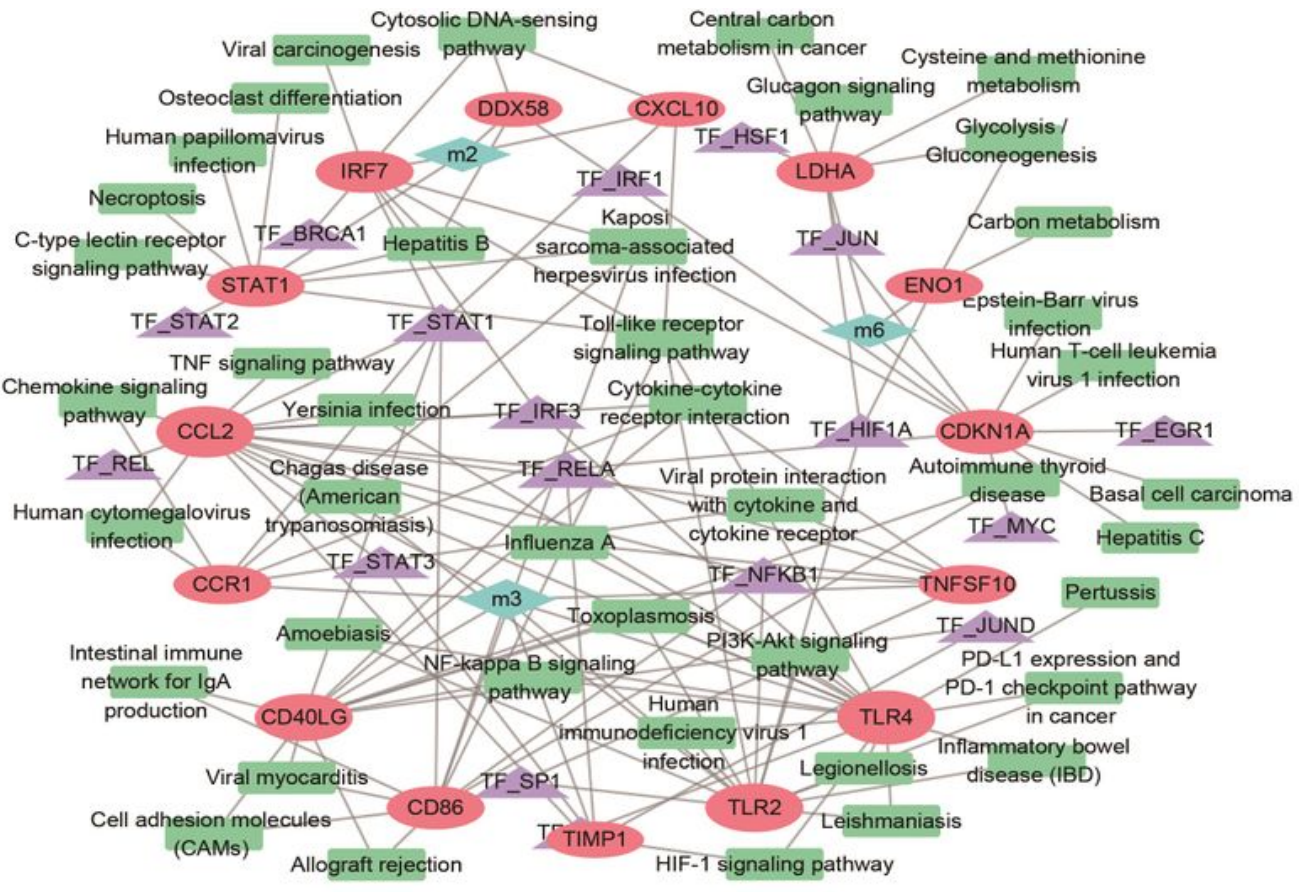

B
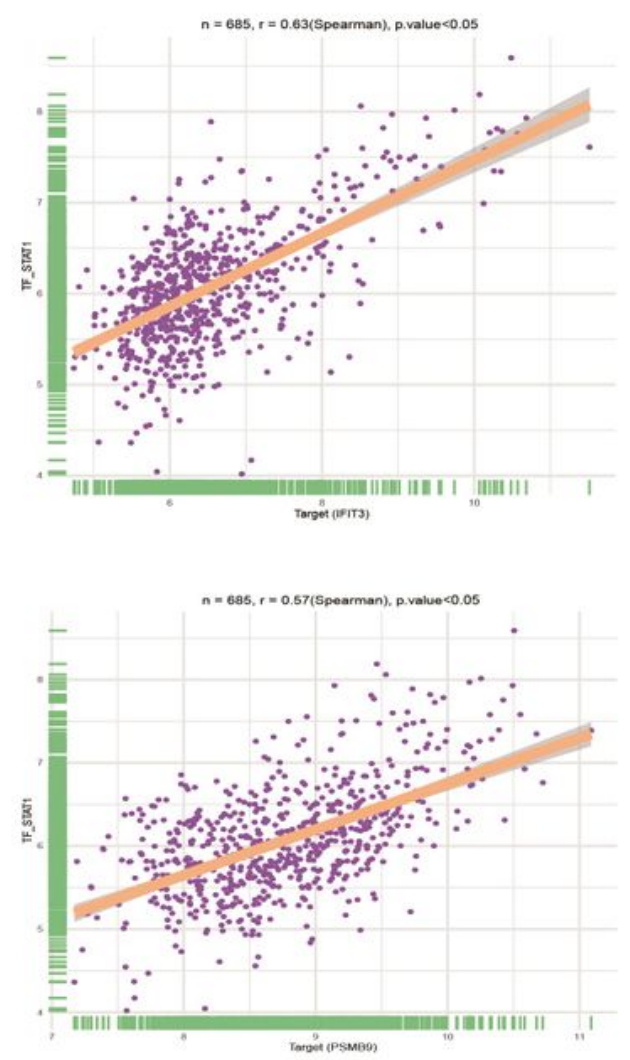
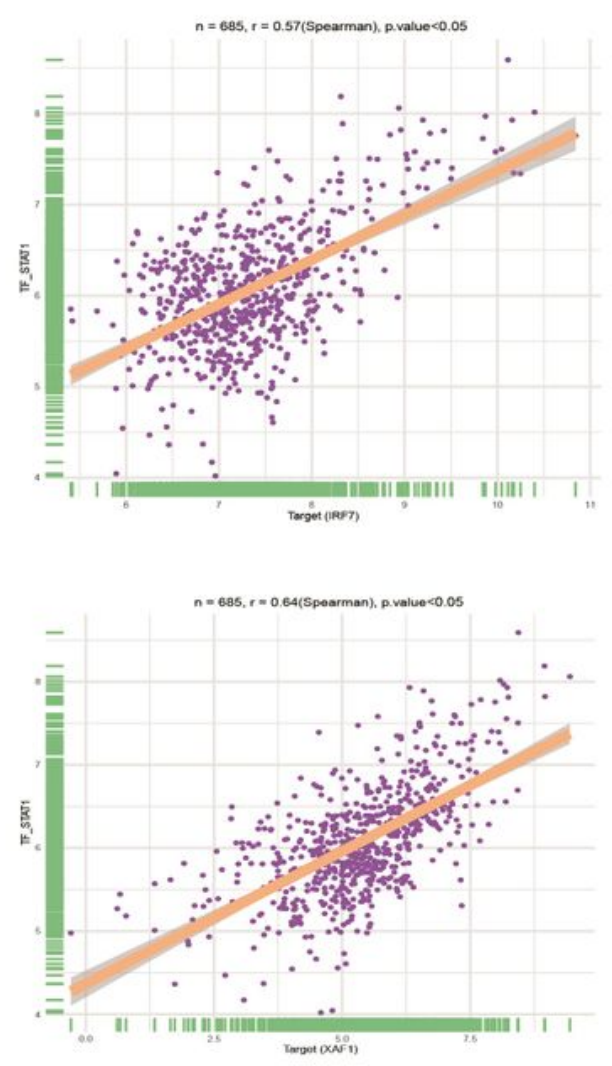

Figure 4 
Identification of transcription regulators. A. Network of transcription factor targeting module gene regulatory signaling pathway. B. Transcription regulators with high correlation with module genes.

\section{Supplementary Files}

This is a list of supplementary files associated with this preprint. Click to download.

- TableS4.xIsx

- TableS3.xIsx

- TableS2.xIsx

- TableS1.xlsx

- FigureS1.tif 